\title{
The use of artificial dermis for corrective surgery on burn scars
}

\author{
Banjerd Praditsuktavorn, Pornprom Muangman \\ Division of Trauma Surgery, Department of Surgery, Faculty of Medicine, Mahidol University, Bangkok \\ 10700, Thailand
}

\begin{abstract}
Background: Hypertrophic scarring from burn injuries on large skin areas is of great concern for both patients and attending physicians because of significant functional and cosmetic impairment. Surgery for scar corrections is challenging because of limitations on the availability of normal tissue. An alternative method for scar correction using artificial dermis is now possible. Artificial dermis can be used in the reconstruction of scars, burn injuries on large skin areas and in patients who do not have sufficient donor sites for skin grafts after scar excisions.

Objective: To report the efficacy of artificial dermis on a 20 years old Thai man with severe scar contracture from burn injuries.

Method: An open-label case study, the severe contracture case underwent scar excision and was applied with artificial dermis.

Result: A 20-year-old man had severe scar contractures on his left arm, forearm, and elbow, and a shearing wound with a scar at his left elbow. Total scar excision was done on his left arm, forearm, elbow, and hand, and then followed by application of artificial dermis on to the wounds. After 2 weeks, neodermis formation was observed, after which an ultrathin split thickness skin graft was applied to the wounds. One month after sugery, the patient could extend his left elbow fully and the cosmetic result is satisfactory.

Conclusion: Use of artificial dermis should be a new alternative modality in improving our strategy in correcting scar issues from burn injuries, especially on large burn scar areas with limited donor sites for skin grafts.
\end{abstract}

Keywords: Artificial dermis, hypertrophic scar, porcine collagen sponge, scar contracture

Hypertrophic scarring following burn injuries is a great concern for patients with deep burn injuries [1]. Raised scars may cause significant functional and cosmetic impairments including pain, which are responsible for a decreased quality of life [2, 3]. The incidence of scars is over $70 \%$ following burns and $40 \%$ of these are hypertrophic [4]. Using skin grafts and flaps in the reconstruction of movement function is one method to treat postburn hypertrophic scars. Artificial dermis is a new modality to solve this problem, especially in patients with large areas of full thickness burn wounds and who do not have sufficient healthy skin for donor site skin grafts [5].

One type of artificial dermis (Pelnac, Gunze Co, Kyoto, Japan) is composed of an outer silicone layer

Correspondence to: Pornprom Muangman, Division of Trauma Surgery, Department of Surgery, Faculty of Medicine, Mahidol University, Bangkok 10700, Thailand. E-mail: pornprom.mua@ mahidol.ac.th, porprom.mu@gmail.com and inner porcine atelocollagen sponge layer. After the implantation of this artificial dermis on a wound, fibroblasts start to infiltrate into the inner collagen pores and new capillaries are formed within 2 to 3 weeks [6]. The new dermal layer (neodermis) is formed with an extracellular matrix, while the outer silicone layer can be removed (after 2 to 3 weeks). This is then followed by ultrathin split thickness skin graft implantation (approximately $0.15 \mathrm{~mm}$ in depth) on top of the neodermal layer. The limited donor site can be harvested many times within a shorter period compared with a regular split thickness skin graft (approximately $0.3 \mathrm{~mm}$ in depth) because the harvested split thickness skin graft is ultrathin. This artificial dermis is useful to surgeons for their procedures in improving hypertrophic scars. In this case report, we describe our experience in the correction of scar contracture using an artificial dermis (porcine collagen sponge) in a postburn patient with hypertrophic scarring. 


\section{Case report}

A 20-year-old Thai man had postburn injuries to $70 \%$ of his total body surface area incurred 2 years previously. There were lesions on his face, arms, trunk, and left leg. He also developed severe scar contractures on his left arm and had an open wound that was approximately $3 \times 3$ centimeters on his elbow over the joint. The extensive scarring resulted in difficulty in movement and flexibility of his elbow joint (Figure 1). We decided to use an artificial dermis, Pelnac (Gunze Co, Kyoto, Japan ), for the reconstruction of his hypertrophic scar because he had limited quality skin graft area for application to the wound area after the hypertrophic scar excision. The removal of scar contractures involved a total excision of scars from his left arm, forearm, elbow, and hand. The depth of the dissection was extended to normal subcutaneous tissue to achieve complete release of the scars. The scars over his left arm, forearm, elbow, and hand were excised deeply through to healthy fatty tissue. Figure 2 shows the 2 pieces of scars with healed shearing wounds over the left elbow joint area.

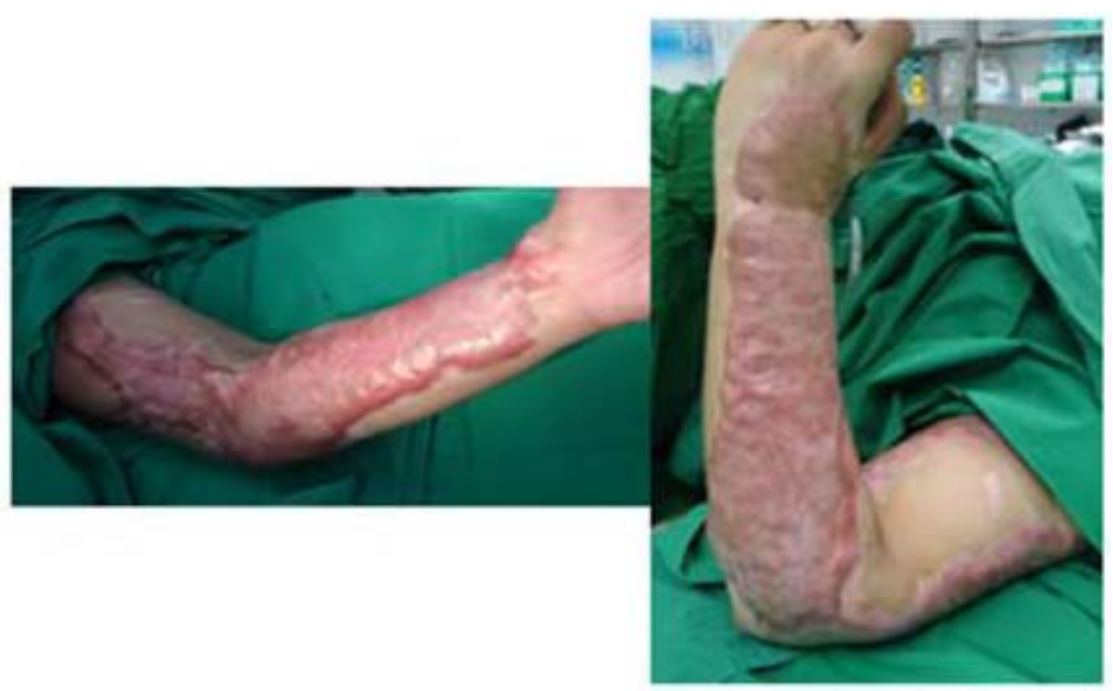

Figure 1. The extensive scarring on the left arm (with permission of the patient).

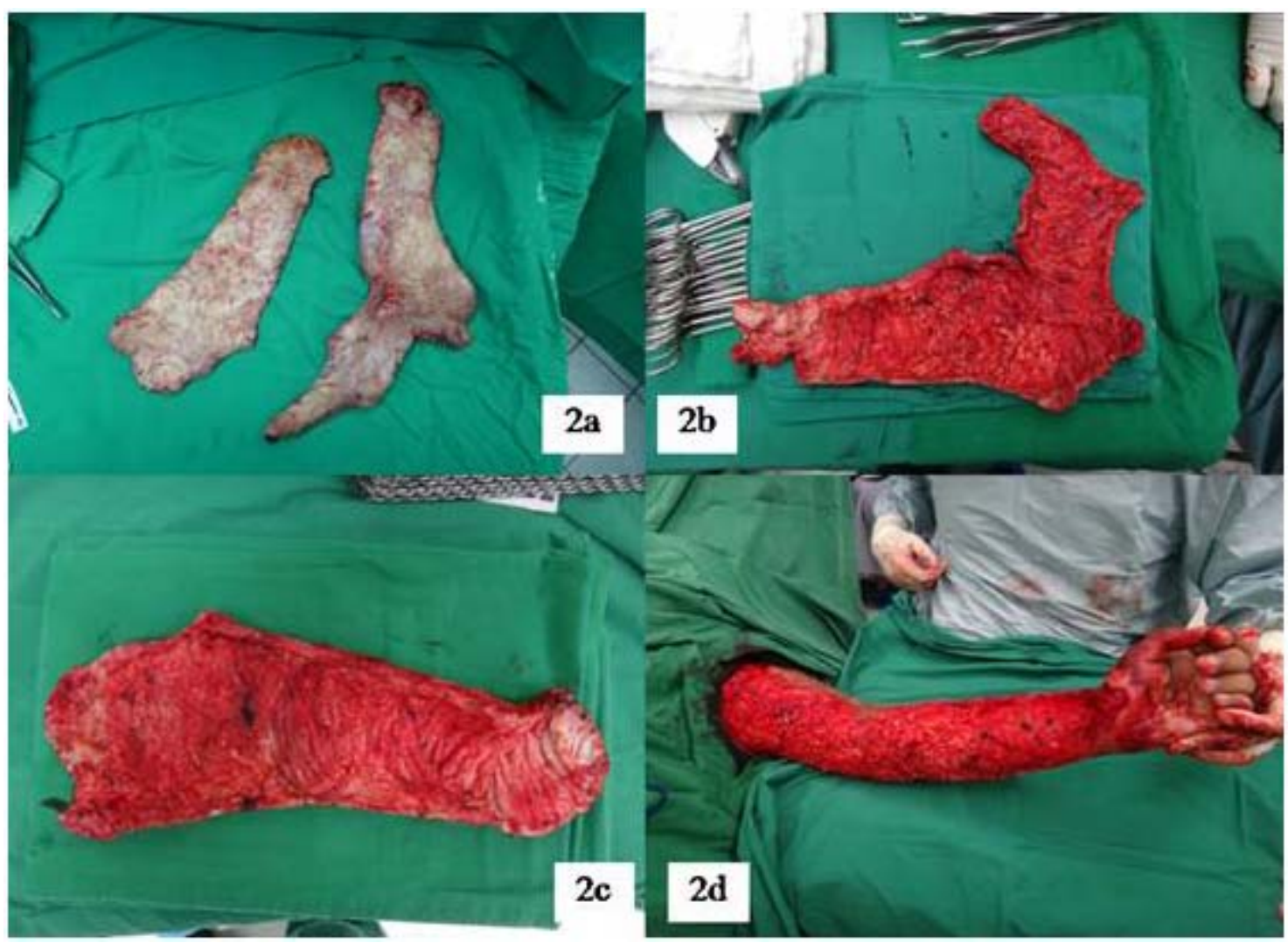

Figure 2. Scar excision from the left arm (with permission of the patient). 
After the artificial dermis was applied onto the wound and stapled to the edges (Figure 3), the wound was covered with a nanocrystalline silver dressing and bandaged with an outer dressing. We changed the outer dressing on postoperative days 3,7 , and 10 , and the patient was transferred to an operating room for removal of staples on day 14 .

Figure 4a illustrates the wound after the artificial dermis was applied for 2 weeks, which demonstrated the complete $(100 \%)$ acceptance of the artificial dermis. The outer silicone sheet layer was then removed (Figure $\mathbf{4 b}$ ) and the neodermal formation was observed underneath (Figure 4c). An ultrathin epidermal skin graft with a thickness of $0.15 \mathrm{~mm}$ was harvested from his thighs using a dermatome (Zimmer, Swindon, UK). The epidermal skin graft was then meshed at a 1:1.5 ratio and then applied onto the artificial dermis template area (Figure 4d). We avoided harvesting a thick skin graft because the patient did not have sufficient healthy skin, and to prevent donor site scarring from harvesting too deeply. The donor site wound healed at day 15 after the surgery. The patient was discharged on day 21 and returned on day 7 , at 1 month, and at 3 months after the surgery. The result was satisfactory. It harmonized with the surrounding normal skin without any complications when observed 3 months after the surgery. The patient could perform an almost complete elbow extension to $170^{\circ}$, compared with $120^{\circ}$ extension before surgery. He also demonstrated no shearing wound, which often occurred over the scar contracture area, especially over joints, after too much extension and movement of his left elbow (Figure 5). Follow up at 1 year showed no recurrent shearing wound over the left elbow joint area (Figure 6).

The patient provided his written informed consent for publication of this case report including the clinical photographs.

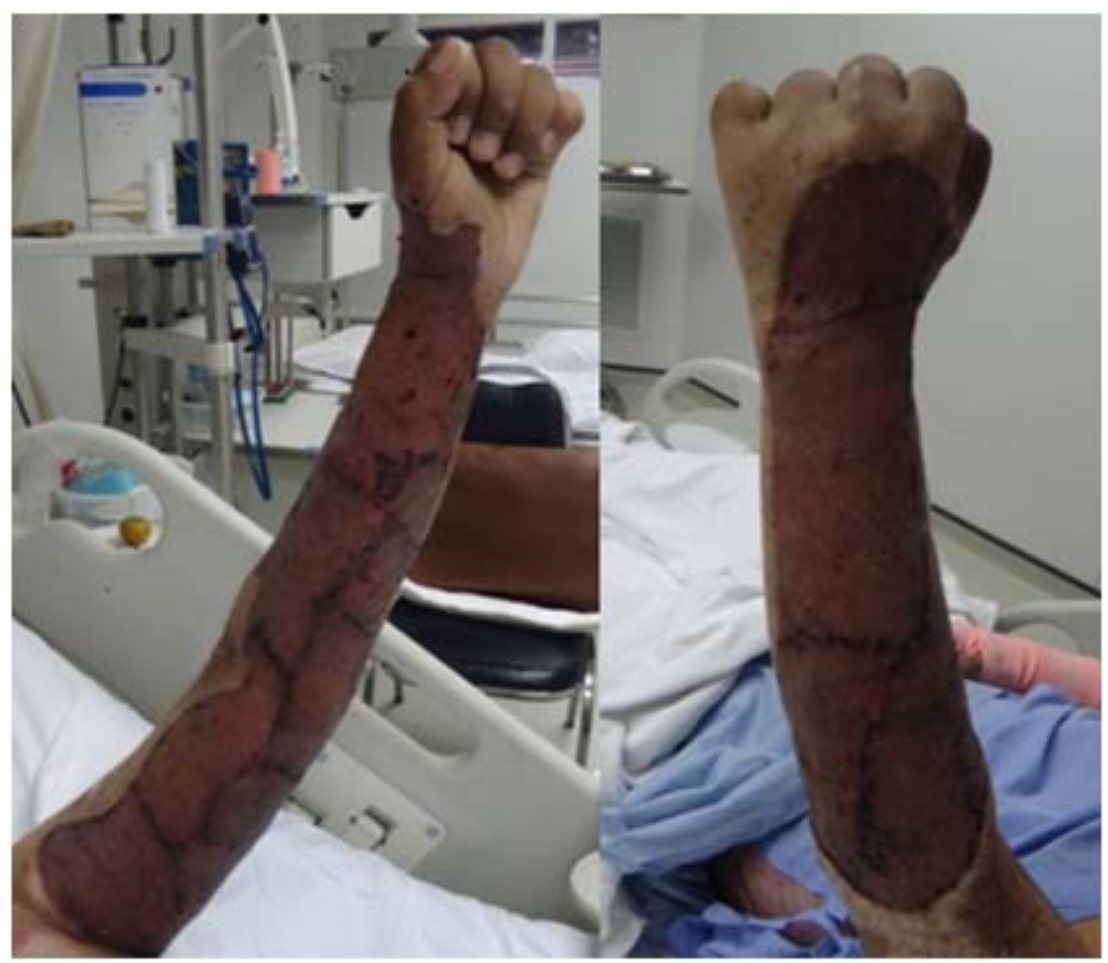

Figure 3. Application of an artificial dermis onto the left arm (with permission of the patient). 


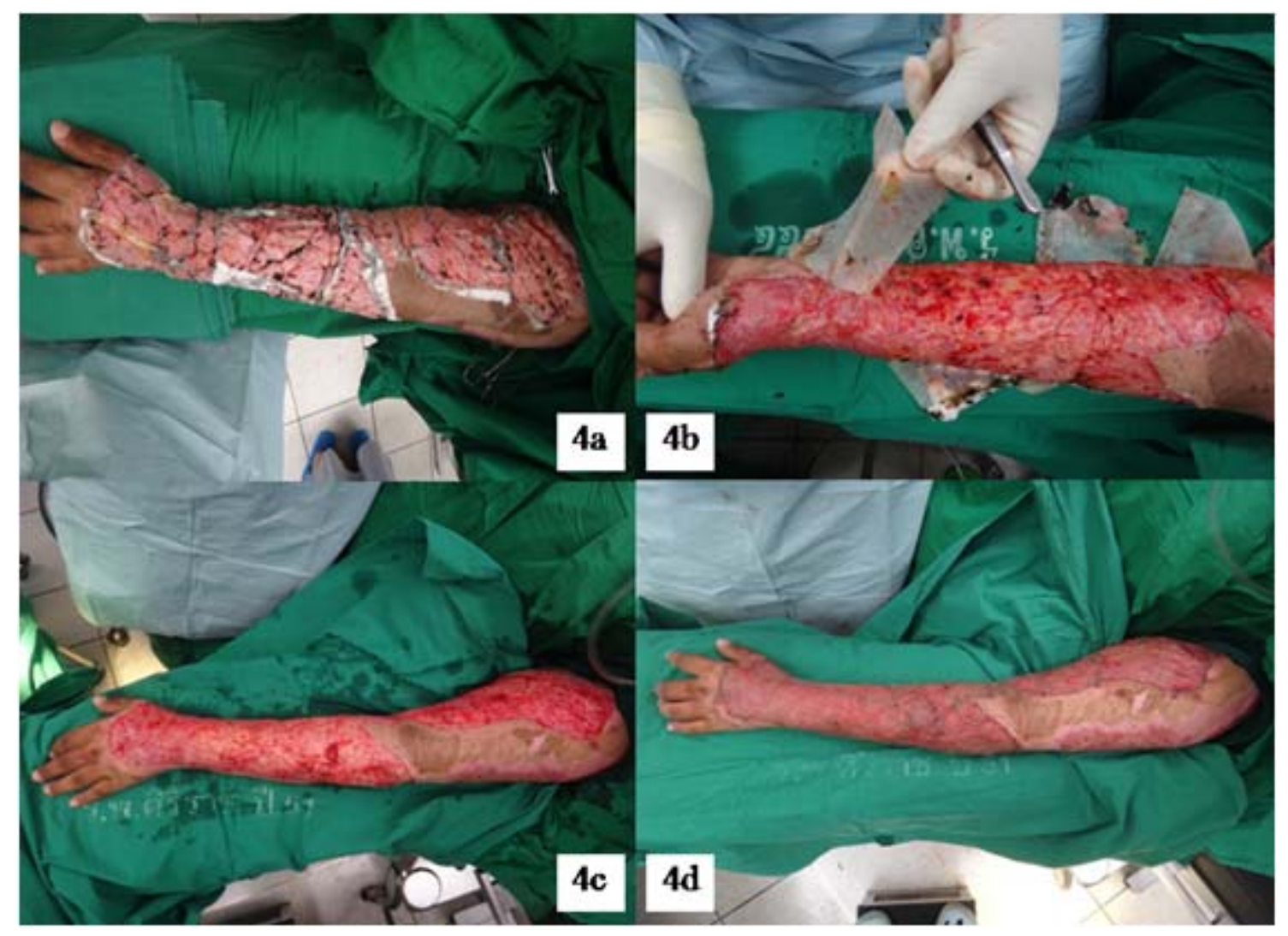

Figure 4 a-d. Removal of silicone layer and epidermal graft application (with permission of the patient).

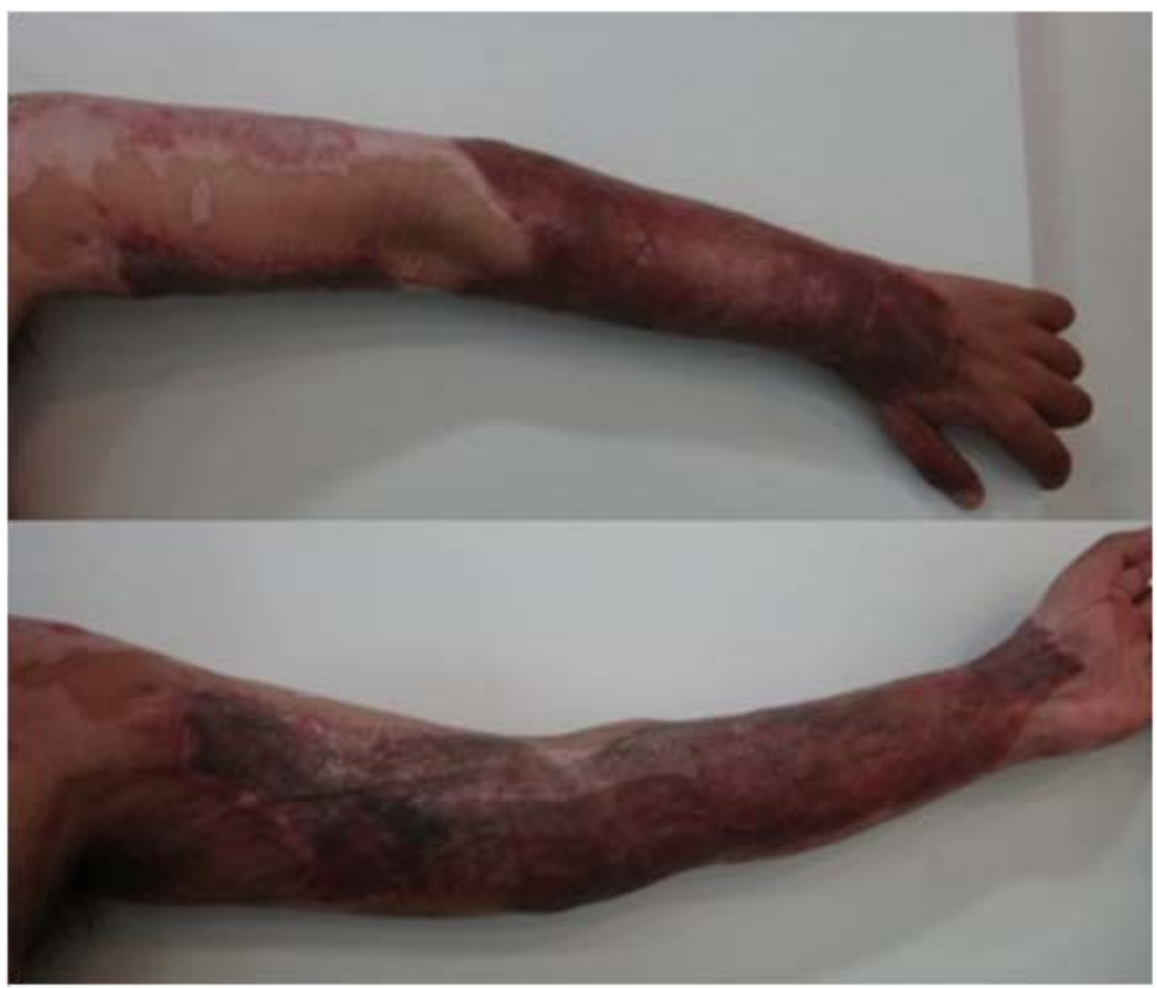

Figure 5. Follow up at 3 months after wound closure (with permission of the patient). 


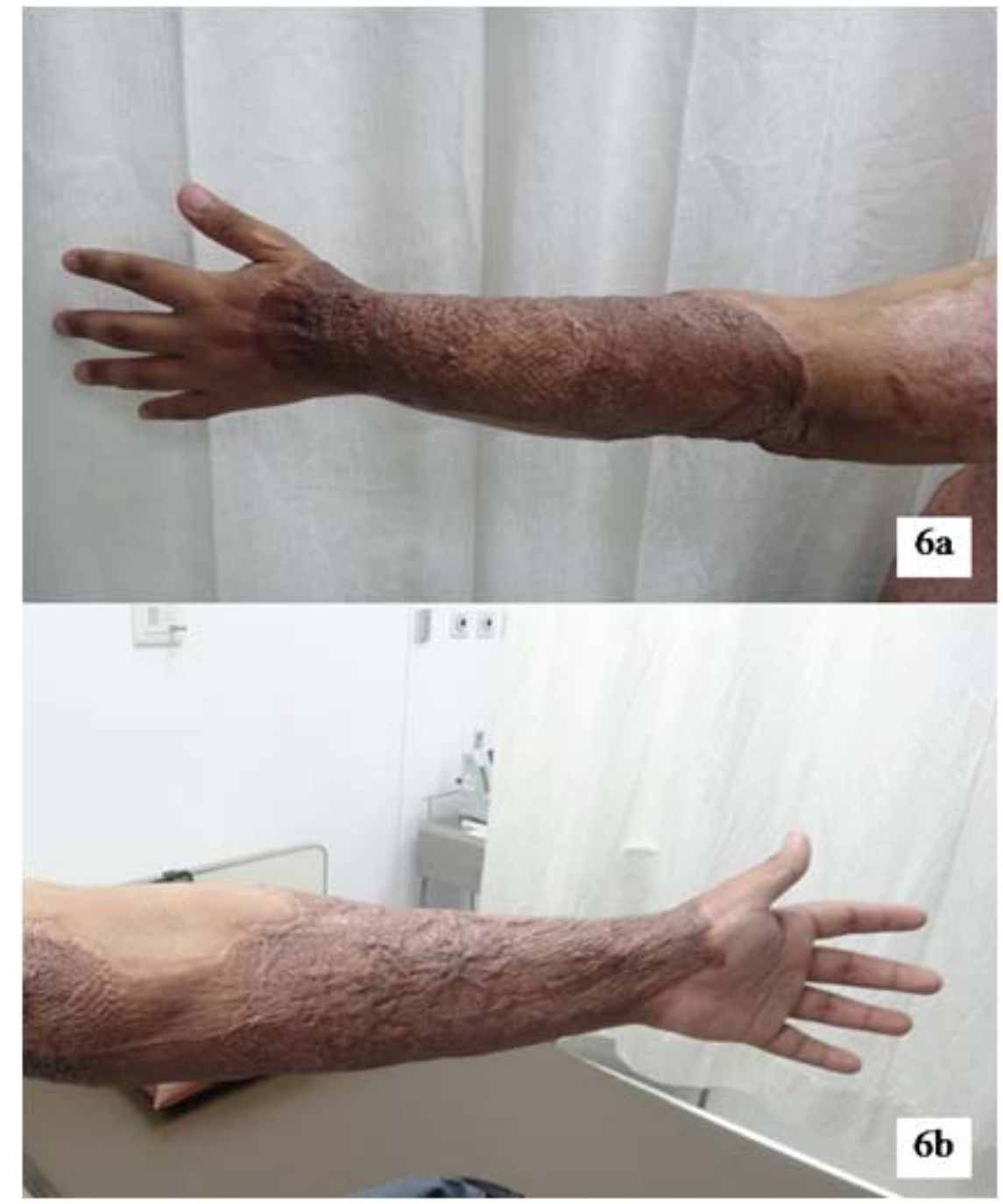

Figure 6. Follow up at 1 year with no recurrent shearing wound over left elbow joint area (a and b) (with permission of the patient).

\section{Discussion}

Scar contracture after burn injuries is challenging. There are several methods by which to prevent and treat hypertrophic scars including topical agents, steroid injections, pressure garments, and surgery. The proper treatment of severe scar contracture is by a surgical procedure to release the skin tension.

One type of artificial dermis (graft for dermal defect) is a bilayer composed of an inner sponge layer of atelocollagen and an outer layer of silicone. It can be used in acute burn treatment and reconstruction procedures, as a single layer in trauma cases, or on other skin loss areas after surgery for tumor or nevus removal. It is very effective in functional and cosmetic improvements [7-10] and postprocedure contracture defects are found to be insignificant [11]. For the present patient, the cosmetic and functional appearance on his left arm was improved. He demonstrated an almost full extension of his left elbow joint compared with before the surgery. We have shown that artificial dermis is beneficial for scar reconstruction; especially when patients have a large burn area and do not have sufficient healthy skin graft area for application to the wound after scar excision. Moreover, this artificial dermis was developed for use in various indications. Growth factor was loaded into the inner layer, which can accelerate chronic wound healing and prolong its stability $[12,13]$. The artificial dermis is impregnated with an antibiotic for application on contaminated wounds [14]. 


\section{Conclusion}

The use of an artificial dermis is effective in surgery for scar contracture. It is an alternative modality in solving scar problems; especially on large areas of burn injuries with a lack of sites for skin graft for wound coverage after scar excision.

\section{Acknowledgments}

Authors acknowledge Mrs. Supaparn Suwannachote and Miss Nantaporn Namviriyachote for their management of this case. We thank the patient for providing his written informed consent for publication of this case report including clinical photographs.

Authors do not have affiliations with or involvement in any organization or entity with any financial interest or nonfinancial interest in this subject matter or materials discussed in this manuscript.

\section{References}

1. Slemp AE, Kirschner RE. Keloids and scars: a review of keloids and scars, their pathogenesis, risk factors, and management. Curr Opin Pediatr. 2006; 18:396-402.

2. Brown BC, McKenna SP, Siddhi K, McGrouther DA, Bayat $\mathrm{A}$. The hidden cost of skin scars: quality of life after skin scarring. J Plast Reconstr Aesthet Surg. 2008; 61:1049-58.

3. Bock O, Schmid-Ott G, Malewski P, Mrowietz U. Quality of life of patients with keloid and hypertrophic scarring. Arch Dermatol Res. 2006; 297:433-8.

4. Gangemi EN, Gregori D, Berchialla P, Zingarelli E, Cairo M, Bollero D, et al. Epidemiology and risk factors for pathologic scarring after burn wounds. Arch Facial Plast Surg. 2008; 10:93-102.

5. Iwuagu FC, Wilson D, Bailie F. The use of skin grafts in post burn contracture release: a 10 year review. Plast Rec Surg. 1999; 103:1198
6. Suzuki S, Matsuda K, Isshiki N, Tamada Y, Ikada Y. Experimental study of a newly developed bilayer artificial skin. Biomaterials. 1990; 11:356-60.

7. Suzuki S, Matsuda K, Isshiki N, Tamada Y, Yoshioka K, Ikada Y. Clinical evaluation of a new bilayer "artificial skin" composed of collagen sponge and silicone layer. British J Plast Surg. 1990; 43:47-54.

8. Groos N, Guillot M, Zilliox R, Braye FM. Use of an artificial dermis (Integra) for the reconstruction of extensive burn scars in children. About 22 Grafts. Eur J Pediatr Surg. 2005; 15:187-92.

9. Soejima K, Nozaki M, Sasaki K, Takeuchi M, Negishi N. Reconstruction of burn deformity using artificialdermis combined with thin split-skin grafting. Burns. 1997; 23:501-4.

10. Suzuki S, Kawai K, Ashoori F, Morimoto N, Nishimura Y, Ikada Y. Long-term follow-up study of artificial dermis composed of outer silicone layer and inner collagen sponge. British J Plast Surg. 2000; 53:659-66.

11. Suzuki S, Matsuda K, Maruguchi T, Nishimura Y, Ikada Y. Further applications of "bilayer artificial skin". Brit J Plast Surg. 1995; 48:222-9.

12. Kawai K, Suzuki S, Tabata Y, Ikada Y, Nishimura Y. Accelerated tissue regeneration through incorporation of basic fibroblast growth factor-impregnated gelatin microspheres into artificial dermis. Biomaterials. 2000; 21:489-99.

13. Morimoto N, Yoshimura K, Niimi M, Ito T, Tada $\mathrm{H}$, Teramukai S, et al. An exploratory clinical trial for combination wound therapy with a novel medical matrix and fibroblast growth factor in patients with chronic skin ulcers: a study protocol. Am J Transl Res. 2012; 4:52-9.

14. Kawai K, Suzuki S, Tabata Y, Taira T, Ikada Y, Nishimura Y. Development of an artificial dermis preparation capable of silver sulfadiazine release. $\mathrm{J}$ Biomed Mater Res. 2001; 57:346-56. 